\title{
SPARSITY BASED SUPER-RESOLUTION OPTICAL IMAGING USING CORRELATION INFORMATION
}

\author{
Oren Solomon $^{\star} \quad$ Maor Mutzafi $\quad$ Mordechai Segev ${ }^{\dagger} \quad$ Yonina C. Eldar ${ }^{\star}$ \\ ${ }^{\star}$ Department of Electrical Engineering, Technion, Haifa 32000, Israel, \\ ${ }^{\dagger}$ Department of Physics and Solid State Institute, Technion, Haifa 32000, Israel.
}

\begin{abstract}
Traditionally, spatial resolution in optical imaging is limited by diffraction. Although sub-wavelength information is absent in the measurements, state-of-the-art fluorescence based localization techniques such as PALM and STORM manage to achieve spatial resolution of tens of nano-meters, but with limited temporal resolution. A more recent technique super-resolution optical fluctuation imaging (SOFI) exploits the temporal statistical behavior of uncorrelated fluorescence emissions to practically improve the spatial resolution by a factor of two over the diffraction limit, but with considerably faster image capturing. Here we propose to exploit the sparse nature of the fluorophores distribution, combined with a statistical prior of uncorrelated emissions such as in SOFI to achieve spatial resolution comparable to PALM/STORM, while retaining the temporal resolution of SOFI. We demonstrate our method on simulations and show improved results over STORM and SOFI. Our method may facilitate super-resolution imaging and capturing of intra-cellular dynamics within living cells.
\end{abstract}

Index Terms - Fluorescence, High-resolution imaging, Compressed sensing, Correlation.

\section{INTRODUCTION}

Spatial resolution in diffractive optical imaging is limited by one half of the optical wavelength; this limit is known as Abbe's diffraction limit [1]. However, modern microscopic methods enable super-resolution, even though information on sub-wavelength features is absent in the measurements. One of the leading subwavelength imaging modalities is based on fluorescence (PALM [2] and STORM [3]). Its basic principle consists of imaging the fluorescent light emitted by fluorophores (point emitters) attached to regions of interest within the sample. PALM and STORM rely on acquiring a sequence of diffraction limited images, such that in each frame only a sparse set of fluorophores are active. The position of each fluorophore is then found through a superlocalization procedure [4]. Subsequent accumulation of singlemolecule localizations result in a grainy high-resolution image, which is then smoothed to form the final super-resolved image. The final image has a spatial resolution of tens of nanometers.

A major disadvantage of these florescence techniques is that they require tens of thousands of exposures, which leads to a long acquisition cycle, typically on the order of several minutes [5]. This implies that fast dynamics - even at the rates of microseconds cannot be captured by PALM/STORM. To reduce acquisition time, an alternative technique named SOFI (super-resolution fluctuation imaging) was proposed [6], which uses high fluorophore density, reducing integration time. In SOFI, the emitters usually overlap in each frame, so that super-localization cannot be performed. However, the emitted photons, which are uncorrelated between different emitters, are registered in consecutive frames, that contain

This work was funded by the European Union's Horizon 2020 research and innovation program under grant agreement No. 646804-ERCCOGBNYQ. information in the pixel-wise temporal correlation between them. The measurements are processed such that correlative information is used, enabling the recovery of features that are smaller than the diffraction limit by a factor of $\sqrt{2}$. By calculating higher order statistics (HOS) in the form of cumulants [7] of each pixel's timetrace, a theoretical resolution increase equal to the square root of the order of the statistics can (in principle) be achieved. Using pixels cross-correlations over time it is possible to increase the resolution gain further, to an overall factor that scales linearly with the order of the statistical calculation [8].

SOFI enables the processing of images with high fluorophore density, thus reducing the number of required frames for image recovery and achieving increased temporal resolution over localization based techniques. However, at least thus far, the spatial resolution offered by SOFI does not reach the level of super-resolution obtained through STORM and PALM, even when using HOS. The use of HOS can in principle increase the spatial resolution, but higher (than the order of two) statistical calculations require an increasingly large number of frames for their estimation, degrading the temporal resolution of SOFI. Moreover, SOFI suffers from a phenomena known as dynamic range expansion, in which weak emitters are masked in the presence of strong ones. The effect is worsened as the statistical order increases.

Achieving super-resolution based on statistical information can be extended beyond the scope of fluorescence microscopy to other imaging modalities. In contrast enhanced ultrasound (CUES), gas micro-bubbles are injected into the bloodstream to image the vascular system. Several localization based super-resolution techniques inspired by STORM and PALM were suggested for CEUS [9], [10]. These methods achieve excellent sub-diffraction spatial resolution but suffer from similar limitations as their optical analogues. Inspired by SOFI, the authors of [11] suggested to exploit the statistical nature of the fluctuations of the micro-bubbles to image capillaries with sub-diffraction resolution while retaining clinically relevant temporal resolution. Such ideas suggest that super-resolution using statistical information can be considered in a wide context of imaging modalities and not only in fluorescence microscopy.

Here we propose a method for super-resolution imaging with short integration time which is also computationally efficient, leading to fast image reconstruction and is suitable for large-scale problems. Our approach enjoys the same benefits of SOFI, i.e. fast frame capturing rate of high fluorophore density frames and the use of correlative information, while offering the possibility of reaching a comparable, single-molecule resolution such as STORM. In fluorescence microscopy we rely on fluorophores which attach only to specific objects of interest. Thus, only sparse areas within the imaged sample emit light. We propose to use sparse recovery on correlation information to achieve recovery with increased spatial resolution, comparable to super-localization methods, while retaining a short temporal acquisition time similar to SOFI. This may facilitate super-resolution imaging of dynamic processes within living cells. We demonstrate these ideas on simulated data and show that our technique overcomes the dynamic range problem of 
SOFI when high-order statistics are used, and results in improved image reconstruction.

Mathematically, our method recovers the support of the emitters, by recovering their variance values. Sparse recovery from correlation information was previously proposed to improve sparse recovery from a small number of measurements [12], [13], [14]. When the non-zero entries of the sparse signal are uncorrelated, support size recovery can be theoretically increased up to $O\left(M^{2}\right)$, where $M$ is the length of a single measurement vector. Here we use similar concepts in order to enhance resolution and improve SNR in optical imaging. Preliminary results were demonstrated in [15]. Here, we provide a detailed mathematical formulation and additional numerical examples.

The rest of the paper is organized as follows: In Section II we present the problem and explain the key idea of SOFI. In Section III we formulate our proposed solution. Simulation results are demonstrated in Section IV.

Throughput the paper, $x$ represents a scalar, $\mathrm{x}$ represents a vector and $\mathbf{X}$ a matrix. The notation $\|\cdot\|_{p}$ represents the standard $p$-norm and $\|\cdot\|_{F}$ the Frobenius norm. Subscript $x_{l}$ denotes the $l$ th element of $\mathbf{x}$ and $\mathbf{x}_{l}$ is the $l$ th column of $\mathbf{X}$.

\section{PROBLEM FORMULATION AND SOFI}

Following [6], [8], the acquired fluorescence signal in the object plane is modeled as a set of $L$ independently fluctuating point sources, with resulting fluorescence source distribution

$$
J(\mathbf{r}, t)=\sum_{k=0}^{L-1} \delta\left(\mathbf{r}-\mathbf{r}_{k}\right) \cdot s_{k}(t) .
$$

Each source (or emitter) has its own time dependent brightness function $s_{k}(t)$, and is located at position $\mathbf{r}_{k} \in \mathbb{R}^{2}, k=$ $0, \ldots, L-1$. The acquired signal in the image plane is the result of the convolution between $J(\mathbf{r}, t)$ and the impulse response of the microscope $u(\mathbf{r})$ (also known as the point spread function (PSF)),

$$
f(\mathbf{r}, t)=\sum_{k=0}^{L-1} u\left(\mathbf{r}-\mathbf{r}_{k}\right) \cdot s_{k}(t) .
$$

Due to the propagation of light, the microscope can be considered as a spatial low-pass filter (LPF) [1], so that (1) consists of frequencies below the cutoff of the PSF. We assume that the measurements are acquired over a period of $t \in[0, T]$. Ideally, our goal is to recover the locations of the emitters, $\mathbf{r}_{k}$ and their variance values with high spatial resolution and short integration time. The final high-resolution image is constructed from the recovered variance value for each emitter.

To proceed, we assume the following:

A 1: The locations $\mathbf{r}_{k}, k=0, \ldots, L-1$ do not depend on time.

$A$ 2: The brightness is uncorrelated in space $E\left\{s_{i}\left(t_{1}\right) s_{j}\left(t_{2}\right)\right\}=$ $0, \forall i \neq j, \forall t_{1}, t_{2}$.

A 3: The brightness functions $s_{k}(t), k=0, \ldots, L-1$ are wide sense stationary with $E\left\{s_{k}(t)\right\}=E_{k}$ and $E\left\{\tilde{s}_{k}(t) \tilde{s}_{k}(t+\tau)\right\}=$ $g_{k}(\tau), \forall \tau$ where $\tilde{s}_{k}(t)=s_{k}(t)-E_{k}$.

In SOFI, the time-trace of each pixel in the captured movie is correlated with itself for some time lag $\tau$. Using assumptions 2 and 3 , the autocorrelation function at each point $\mathbf{r}$ is therefore,

$$
G_{f}(\mathbf{r}, \tau)=\sum_{k=0}^{L-1} u^{2}\left(\mathbf{r}-\mathbf{r}_{k}\right) \cdot g_{k}(\tau) .
$$

Usually the zero time-lag is used and the final SOFI image is the value of $G_{f}(\mathbf{r}, 0)$ at each point $\mathbf{r}$, where $g_{k}(0)$ represents the variance of emitter $s_{k}$. We can see from (2) that the autocorrelation function depends on the PSF squared. If the PSF is assumed to be Gaussian, its width is reduced by a factor of $\sqrt{2}$. However, the final SOFI image retains the same low resolution grid as the captured movie. Similar statistical calculations can be performed for adjacent pixels in the movie leading to a simple interpolation grid with increased number of pixels in the high-resolution image, but at the cost of increased statistical order using cumulants [7]. Higher order statistics reduce the PSF size further but at the expense of degraded SNR and dynamic range for a given number of frames [8].

In the next section we introduce our sparsity based method. We rely on correlations only without resorting to higher order statistics, thus maintaining a short acquisition time, similar to correlationbased SOFI. In contrast to SOFI, we exploit the sparse nature of the emitter's distribution and recover a high-resolution image on a much denser grid than the camera's grid. This leads to spatial super-resolution without the need to perform interpolation using higher order statistics [8]. Our approach is based on Fourier domain analysis, and can be implemented very efficiently.

Since we rely on the assumption of a sparse distribution of emitters, we emphasize that it is far less restrictive than in PALM / STORM. In particular we allow for much higher density of fluorophores, as long as the features we wish to recover are spatially sparse. The assumption of uncorrelated emissions provides further prior information to exploit, while the correlation domain provides more effective measurements.

\section{SPARSE FOURIER SOFI}

To increase resolution by exploiting sparsity, we start by introducing a Cartesian sampling grid with spacing $\Delta_{L}$, which we refer to as the low-resolution grid. The low-resolution signal (1) can be expressed over this grid as

$$
\begin{aligned}
& f\left[m \Delta_{L}, n \Delta_{L}, t\right]= \\
& \sum_{k=0}^{L-1} u\left[m \Delta_{L}-m_{k}, n \Delta_{L}-n_{k}\right] s_{k}(t), m, n=[0, \ldots, M-1],
\end{aligned}
$$

where $\mathbf{r}_{k}=\left[m_{k}, n_{k}\right]^{T} \in \mathbb{R}^{2}$. We discretize the possible locations of the emitters $\mathbf{r}_{k}$, over a discrete Cartesian grid $i, l=0, \ldots, N-1$, $L \ll N$ with resolution $\Delta_{h}$, such that $\left[m_{k}, n_{k}\right]=\left[i_{k}, l_{k}\right] \Delta_{h}$ for some integers $i_{k}, l_{k} \in[0, \ldots, N-1]$. We refer to this grid as the high-resolution grid.

The latter discretization implies that (3) is sampled (spatially) over a grid of size $M \times M$, while the emitters reside on a grid of size $N \times N$, with the $i$ th pixel having a fluctuation function $s_{i l}(t)$ (only $L$ such pixels actually contain fluctuating emitters, according to (3)). If there is no emitter in the $i l$ 'th pixel, then $s_{i l}(t)=0$ for all $t$. We further assume that the PSF $u$ is known.

Rewriting (3) in Cartesian form with respect to the grid of emitters yields,

$$
\begin{aligned}
& f\left[m \Delta_{L}, n \Delta_{L}, t\right]= \\
& \quad \sum_{i=0}^{N-1} \sum_{l=0}^{N-1} u\left[m \Delta_{L}-i \Delta_{h}, n \Delta_{L}-l \Delta_{h}\right] s_{i l}(t) .
\end{aligned}
$$

For simplicity we assume that $\Delta_{L}=P \Delta_{h}$ for some $P \geq 1$, and consequently $N=P M$. In addition, it holds that $m \Delta_{L}-i \Delta_{h}=$ $(m P-i) \Delta_{h}$. Omitting the spacing $\Delta_{h}$, we can rewrite (4) as

$$
f[m P, n P, t]=\sum_{i, l=0}^{N-1} u[m P-i, n P-l] s_{i l}(t) .
$$

We now what to present (5) in the Fourier domain, which will lead to an efficient implementation of our method.

Since $f[m P, n P, t]$ is an $M \times M$ sequence, denote by $y[m, n, t]=f[m P, n P, t]$ and its $M \times M$ two dimensional discrete 
Fourier transform (DFT), by $Y\left[k_{m}, k_{n}, t\right]$. Performing an $M \times M$ two dimensional DFT on $y[m, n, t]$ yields

$$
\begin{aligned}
Y\left[k_{m}, k_{n}, t\right] & =\sum_{m, n=0}^{M-1} f[m P, n P, t] e^{-j \frac{2 \pi}{M} k_{m} m} e^{-j \frac{2 \pi}{M} k_{n} n} \\
= & \sum_{\substack{i, l=0 \\
M P-P}}^{N-1} s_{i l}(t) . \\
& \sum_{\hat{m}, \hat{n}=0, P, \ldots} u[\hat{m}-i, \hat{n}-l] e^{-j \frac{2 \pi}{M P} k_{m} \hat{m}} e^{-j \frac{2 \pi}{M P} k_{n} \hat{n}},
\end{aligned}
$$

where we defined $\hat{m}=m P$ and $\hat{n}=n P$ and $k_{m}, k_{n}=$ $0, \ldots, M-1$. Next, consider $\hat{m}, \hat{n}=0, \ldots, N-1$ and define the $N \times N$ sequence,

$$
\tilde{u}[\hat{m}, \hat{n}]= \begin{cases}u[\hat{m}, \hat{n}], & \hat{m}, \hat{n}=0, P, \ldots, N-P, \\ 0, & \text { else, }\end{cases}
$$

where $u$ is the discretized PSF sampled over $M \times M$ points of the low-resolution grid. Thus, we can equivalently write for $Y\left[k_{m}, k_{n}, t\right]$,

$$
\begin{aligned}
& Y\left[k_{m}, k_{n}, t\right]=\sum_{i, l=0}^{N-1} s_{i l}(t) . \\
& \sum_{\hat{m}, \hat{n}=0}^{N-1} \tilde{u}[\hat{m}-i, \hat{n}-l] e^{-j \frac{2 \pi}{N} k_{m} \hat{m}} e^{-j \frac{2 \pi}{N} k_{n} \hat{n}} .
\end{aligned}
$$

By defining $p=\hat{m}-i$ and $q=\hat{n}-l$, (7) becomes

$$
Y\left[k_{m}, k_{n}, t\right]=\tilde{U}\left[k_{m}, k_{n}\right] \sum_{i, l=0}^{N-1} s_{i l}(t) e^{-j \frac{2 \pi}{N} k_{m} i} e^{-j \frac{2 \pi}{N} k_{n} l}
$$

with

$$
\tilde{U}\left[k_{m}, k_{n}\right]=\sum_{p, q=0}^{N-1} \tilde{u}[p, q] e^{-j \frac{2 \pi}{N} k_{m} p} e^{-j \frac{2 \pi}{N} k_{n} q} .
$$

Note that $\tilde{U}\left[k_{m}, k_{n}\right]$ is the $N \times N$ two dimensional DFT of the $N \times N$ sequence $\tilde{u}$, evaluated at discrete frequencies $k_{m}, k_{n}=$ $0, \ldots, M-1$.

From (6) and (9), it holds that $\tilde{U}\left[e^{-j \frac{2 \pi}{N} k_{m}}, e^{-j \frac{2 \pi}{N} k_{n}}\right]=$ $U\left[e^{-j \frac{2 \pi}{M} k_{m}}, e^{-j \frac{2 \pi}{M} k_{n}}\right]$ for $k_{m}, k_{n}=0, \ldots, M-1(N=P M)$, where $U$ is the $M \times M$ two dimensional DFT of $u$ sampled on the low-resolution grid.

Denote the column-wise stacking of each frame $Y\left[k_{m}, k_{n}, t\right]$ as an $M^{2}$ long vector $\mathbf{y}(t)$ and in a similar manner, $\mathbf{s}(t)$ is a length$N^{2}$ vector stacking of $s_{i l}(t)$ for all $i l$. We also define the $M^{2} \times$ $M^{2}$ diagonal matrix, $\mathbf{H}=\operatorname{diag}\{\tilde{U}[0,0], \ldots, \tilde{U}[M-1, M-1]\}$. Vectorizing (8) then yields

$$
\mathbf{y}(t)=\mathbf{H}\left(\mathbf{F}_{M} \otimes \mathbf{F}_{M}\right) \mathbf{s}(t)=\mathbf{A} \mathbf{s}(t), \quad \mathbf{A} \in \mathbb{C}^{M^{2} \times N^{2}},
$$

where $\mathbf{s}(t)$ is an $L$-sparse vector and $\mathbf{F}_{M}$ denotes a partial $M \times N$ DFT matrix (its $M$ rows are the corresponding $M$ low frequency rows from a full $N \times N$ discrete Fourier matrix). Using assumption A 3 , we define the autocorrelation matrix of $\mathbf{y}(t)$ as

$$
\mathbf{R}_{y}(\tau)=E\left\{(\mathbf{y}(t)-E\{\mathbf{y}(t)\})(\mathbf{y}(t+\tau)-E\{\mathbf{y}(t+\tau)\})^{H}\right\} .
$$

For a discrete time-lag $\tau$ and total number of frames $T, \mathbf{R}_{y}(\tau)$ is estimated from the movie frames using the empirical correlation

$$
\mathbf{R}_{y}(\tau)=\frac{1}{T-\tau} \sum_{t=1}^{T-\tau}(\mathbf{y}(t)-\overline{\mathbf{y}})(\mathbf{y}(t+\tau)-\overline{\mathbf{y}})^{H},
$$

with

$$
\overline{\mathbf{y}}=\frac{1}{T} \sum_{t=1}^{T} \mathbf{y}(t)
$$

From (10),

$$
\mathbf{R}_{y}(\tau)=\mathbf{A R}_{s}(\tau) \mathbf{A}^{H},
$$

for some time-lag $\tau$. Under assumption $\mathrm{A} 2, \mathbf{R}_{s}(\tau)$ is a diagonal matrix. Therefore, (12) can be written as

$$
\mathbf{R}_{y}(\tau)=\sum_{l=1}^{N^{2}} \mathbf{a}_{l} \mathbf{a}_{l}^{H} r_{s_{l}}(\tau),
$$

with $\mathbf{a}_{l}$ being the $l$ th column of $\mathbf{A}, \mathbf{r}_{s}(\tau)=\operatorname{diag}\left\{\mathbf{R}_{s}(\tau)\right\}$ and $r_{s_{l}}(\tau)$ the $l$ th entry of $\mathbf{r}_{s}(\tau)$. By taking $\tau=0$ we estimate the variance of $s_{i j}(t), i, j=0, \ldots, N-1$ (as written in assumption A 3), but it is also possible to take into account the fact that the autocorrelation matrix $\mathbf{R}_{y}(\tau)$ may be non-zero for $\tau \neq 0$. For simplicity we use $\tau=0$. The support of $\mathbf{r}_{s}(\tau)$ is equivalent to the support of $\mathbf{s}(t)$, which in turn is equivalent to the locations of the emitters on a grid with spacing $\Delta_{h}$. Thus, our problem reduces to recovering the $L$ non-zero values of $r_{s_{l}}(0)$ in (13).

We denote $\mathbf{x}=\mathbf{r}_{s}(0)$ and use the LASSO formulation [16] to construct the following convex optimization problem

$$
\min _{\mathbf{x} \geq \mathbf{0}} \lambda\|\mathbf{x}\|_{1}+\frac{1}{2}\left\|\mathbf{R}_{y}(0)-\sum_{l=1}^{N^{2}} \mathbf{a}_{l} \mathbf{a}_{l}^{H} x_{l}\right\|_{F}^{2},
$$

with a regularization parameter $\lambda \geq 0$ and $x_{l}$ denoting the $l$ th entry in $\mathbf{x}$. We note that it is possible to write a similar formulation to (F-LASSO), accounting also for $\tau>0$ (without the non-negativity constraint).

We solve (F-LASSO) iteratively using the FISTA algorithm [17], [18], [19], which at each iteration performs a gradient step and then a thresholding step. By performing the calculations in the DFT domain, we can calculate the gradient of the smooth part of (FLASSO), that is the squared Frobenius norm, very efficiently. Our technique is summarized in Algorithm 1.

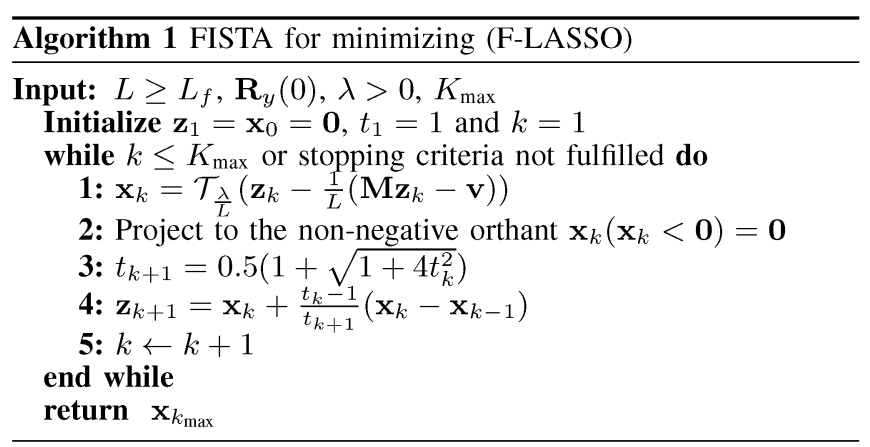

\section{SIMULATIONS AND RESULTS}

We numerically simulated a movie of sub-wavelength features over 1000 frames with some additional out-of-focus features and Gaussian noise with SNR $=14.95 \mathrm{~dB}$, defined as

$$
\mathrm{SNR}=20 \cdot \log _{10} \frac{\left\|\mathbf{Y}_{\text {movie }}\right\|_{F}}{\left\|\mathbf{N}_{\text {movie }}\right\|_{F}}
$$

were $\mathbf{Y}_{\text {movie }}$ is an $M^{2} \times T$ matrix, representing the entire blurred, noise free movie (each movie frame is column stacked as a single column in $\mathbf{Y}_{\text {movie }}$ ) and $\mathbf{N}_{\text {movie }}$ is the added noise to all the frames (same dimensions as $\mathbf{Y}_{\text {movie }}$ ). 

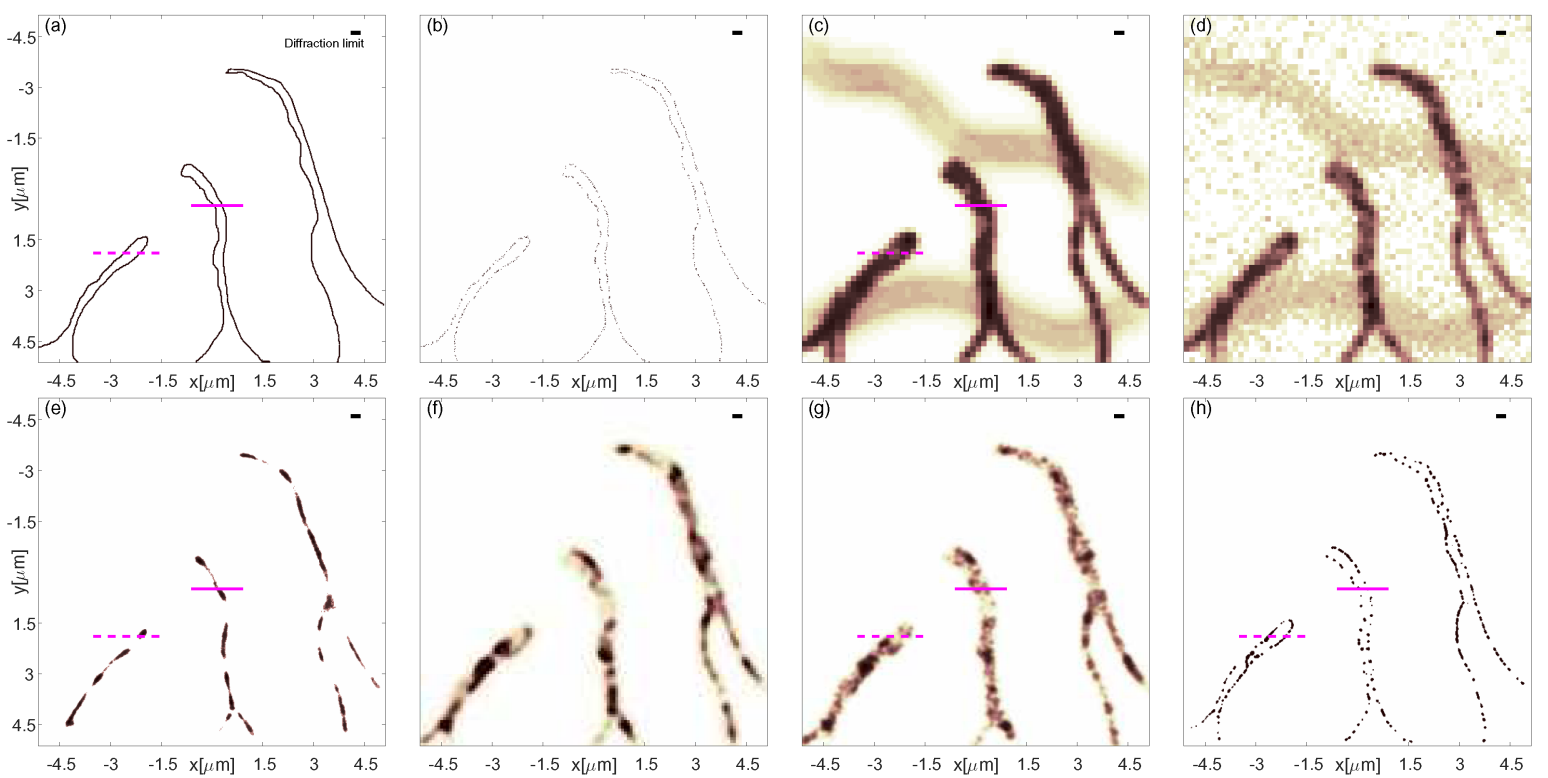

Fig. 1: Upper row: unprocessed data. (a) Ground truth: high resolution image of simulated sub-wavelength features. (b) Positions of emitters in a single frame. (c) Diffraction-limited image. (d) Single diffraction limited frame. Lower row: recovered images from a noisy sequence of 1000 frames. (e) Smoothed ThunderSTORM (f) Correlations SOFI (zero time-lag). (g) $4^{\text {th }}$ order SOFI (in absolute value, zero time-lag). (h) Our sparsity based method. The figures were contrast enhanced slightly for display purposes only.

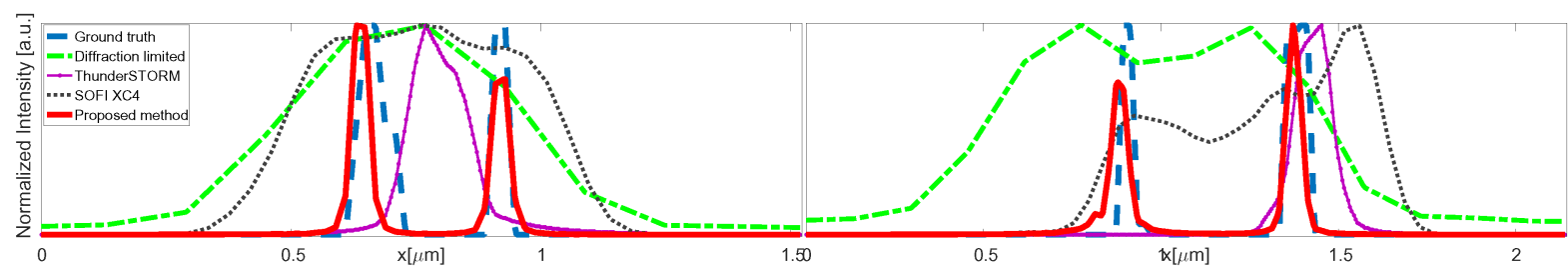

Fig. 2: Normalized cross-sections along the solid line (left) and the dashed line (Right) of Fig. 1, comparing the ground truth (dashed blue, Fig. 1a), diffraction-limited image (dash dot green, Fig. 1c), smoothed ThunderSTORM (solid thin purple, Fig. 1e), $4^{\text {th }}$ order SOFI (black dot, Fig. 1g), and our sparsity based method (solid red, Fig. 1h).

In Figure 1a we show the simulated ground truth of the image with subwavelength features of size $512 \times 512$ pixels. The imaging wavelength is $800 \mathrm{~nm}$ with a numerical aperture of 1.4. Figure $1 \mathrm{~b}$ shows the positions of the emitters for the first frame in the movie, while Fig. 1c shows the diffraction limited image (a sum of all 1000 frames). Figure 1d shows a single frame from the simulated movie, where each frame size is $64 \times 64$ pixels and the pixel size corresponds to $160 \mathrm{~nm}$. The PSF was generated using the freely available PSF generator [20], [21]. Figure 1e shows smoothed ThunderSTORM [22] reconstruction (freely available code). Since the ground truth is of size $512 \times 512$ pixels, the raw localizations image was resized to that size and smoothed with a Gaussian kernel. Figures $1 \mathrm{f}$ and $1 \mathrm{~g}$ show the second and forth order SOFI images respectively (absolute values, zero time-lag). SOFI reconstructions were performed using the freely available code of bSOFI [23], which also includes a Richardson-Lucy deconvolution step with the discretized PSF used in our method. Last, Fig. 1h displays the reconstruction of our method $(512 \times 512$ pixels $)$ after smoothing with the same kernel used in Fig. 1e.

In Fig. 2 left and 2 right we show selected intensity crosssections along two lines. Our method reconstructs images of higher resolution with more details compared to the ThunderSTORM and SOFI images.

Figures 1 and 2 demonstrate that our approach achieves increased resolution and additional details over existing methods, when high labeling density is used, and manages to detect the cavi- ties within the sub-wavelength features which are absent in the low resolution movie and ThunderSTROM and SOFI reconstructions.

\section{CONCLUSIONS}

We proposed a method which improves the spatial resolution of SOFI. Our approach exploits both the sparse nature of the emitters and their uncorrelated emissions to facilitate reconstruction with a spatial resolution comparable to STORM, while retaining the temporal resolution of SOFI. We compared our reconstruction to both STORM and SOFI reconstructions on simulated data, achieving preferable results in terms of support detection and separation of sub-diffraction features. Similar concepts also apply to other imaging modalities, such as CEUS scans. We believe that these improvements may facilitate super-resolution imaging of dynamic processes within living cells and that the proposed framework can be extended to a much wider range of imaging modalities, as long as there are statistical priors which can be exploited alongside sparsity.

\section{ACKNOWLEDGMENT}

We thank Prof. Shimon Weiss and Xiyu Yi for fruitful discussions on SOFI and super-resolution fluorescence microscopy.

\section{REFERENCES}

[1] M. Born and E. Wolf, Principles of optics: electromagnetic theory of propagation, interference and diffraction of light. CUP Archive, 2000. 
[2] E. Betzig, G. H. Patterson, R. Sougrat, O. W. Lindwasser, S. Olenych, J. S. Bonifacino, M. W. Davidson, J. LippincottSchwartz, and H. F. Hess, "Imaging intracellular fluorescent proteins at nanometer resolution.," Science (New York, N.Y.), vol. 313, no. 5793, pp. 1642-1645, 2006.

[3] M. J. Rust, M. Bates, and X. Zhuang, "Sub-diffractionlimit imaging by stochastic optical reconstruction microscopy (STORM).," Nature methods, vol. 3, no. 10, pp. 793-795, 2006.

[4] A. Small and S. Stahlheber, "Fluorophore localization algorithms for super-resolution microscopy," Nature methods, vol. 11, no. 3, pp. 267-279, 2014.

[5] M. J. Rust, M. Bates, and X. Zhuang, "Localization accuracy of single switches before and after drift correction. Supplementary Material: Sub-diffraction-limit imaging by stochastic optical reconstruction microscopy (STORM).," p. 29.

[6] T. Dertinger, R. Colyer, G. Iyer, S. Weiss, and J. Enderlein, "Fast, background-free, 3D super-resolution optical fluctuation imaging (SOFI).," Proceedings of the National Academy of Sciences of the United States of America, vol. 106, no. 52, pp. 22287-22292, 2009.

[7] J. M. Mendel, "Tutorial on higher-order statistics (spectra) in signal processing and system theory: theoretical results and some applications," Proceedings of the IEEE, no. 3, pp. 278305.

[8] T. Dertinger, R. Colyer, R. Vogel, J. Enderlein, and S. Weiss, "Achieving increased resolution and more pixels with Superresolution Optical Fluctuation Imaging (SOFI).," Optics express, vol. 18, no. 18, pp. 18875-18885, 2010.

[9] Y. Desailly, O. Couture, M. Fink, and M. Tanter, "Sono-activated ultrasound localization microscopy," Applied Physics Letters, vol. 103, no. 17, p. 174107, 2013.

[10] C. Errico, J. Pierre, S. Pezet, Y. Desailly, Z. Lenkei, O. Couture, and M. Tanter, "Ultrafast ultrasound localization microscopy for deep super-resolution vascular imaging," Nature, vol. 527, no. 7579, pp. 499-502, 2015.

[11] A. Bar-Zion, C. Tremblay-Darveau, O. Solomon, D. Adam, and Y. Eldar, "Fast vascular ultrasound imaging with enhanced spatial resolution and background rejection," IEEE Transactions on Medical Imaging, 2016.

[12] P. Pal and P. P. Vaidyanathan, "Pushing the Limits of Sparse Support Recovery Using Correlation Information," vol. 63, no. 3, pp. 711-726, 2015.

[13] Y. C. Eldar, Sampling Theory: Beyond Bandlimited Systems. Cambridge University Press, 2015.

[14] D. Cohen and Y. C. Eldar, "Sub-Nyquist Sampling for Power Spectrum Sensing in Cognitive Radios: A Unified Approach," IEEE Transactions on Signal Processing, no. 15, pp. 1-1.

[15] O. Solomon, M. Mutzafi, X. Yi, S. Weiss, Y. C. Eldar, and M. Segev, "Sparsity-based super-resolution optical fluctuation imaging," in CLEO: Applications and Technology, pp. AM4O-4, Optical Society of America, 2016.

[16] R. Tibshirani, "Regression shrinkage and selection via the lasso," Journal of the Royal Statistical Society. Series B (Methodological), pp. 267-288, 1996.

[17] J. Friedman, T. Hastie, and R. Tibshirani, The elements of statistical learning, vol. 1. Springer series in statistics Springer, Berlin, 2001

[18] A. Beck and M. Teboulle, "A Fast Iterative ShrinkageThresholding Algorithm," SIAM Journal on Imaging Sciences, vol. 2, no. 1, pp. 183-202, 2009

[19] T. Wimalajeewa, Y. C. Eldar, and P. K. Varshney, "Recovery of Sparse Matrices via Matrix Sketching," ArXiv, vol. 2, no. 5, pp. 1-5, 2013.

[20] H. Kirshner, F. Aguet, D. Sage, and M. Unser, "3-d psf fitting for fluorescence microscopy: implementation and localization application," Journal of microscopy, vol. 249, no. 1, pp. 13$25,2013$.
[21] H. Kirshner, D. Sage, and M. Unser, " $3 \mathrm{~d}$ psf models for fluorescence microscopy in imagej," in Proceedings of the Twelfth International Conference on Methods and Applications of Fluorescence Spectroscopy, Imaging and Probes (MAF'11), p. 154, 2011.

[22] M. Ovesnỳ, P. Křížek, J. Borkovec, Z. Švindrych, and G. M. Hagen, "Thunderstorm: a comprehensive imagej plug-in for palm and storm data analysis and super-resolution imaging," Bioinformatics, vol. 30, no. 16, pp. 2389-2390, 2014.

[23] S. Geissbuehler, N. L. Bocchio, C. Dellagiacoma, C. Berclaz, M. Leutenegger, and T. Lasser, "Mapping molecular statistics with balanced super-resolution optical fluctuation imaging (bsofi)," Optical Nanoscopy, vol. 1, no. 1, p. 1, 2012. 